\title{
Aplicação de realidade virtual para projeto de inspeção de bioincrustação em casco de navio
}

\author{
Danilo Duarte Lisboa ${ }^{*, * *}$ Arthur Oliveira Viana ${ }^{*, * *}$ \\ Rodrigo Souza Guedes, ${ }^{* * *}$ Ana Letícia Pilz de Castro*** \\ Philip von Pritzelwitz ${ }^{* *}$ \\ Paulo Henrique Vieira Magalhães ${ }^{* * * *}$
* Departamento de Engenharia de Controle e Automação, Universidade Federal de Ouro Preto, MG (e-mails: danilo.lisboa@aluno.ufop.edu.br, arthur.viana@aluno.ufop.edu.br,rodrigo.guedes@aluno.ufop.edu.br) ** Instituto Tecnológico Vale, MG (e-mail: philip.pritzelwitz@itv.org) *** Departamento de Engenharia Civil, Universidade Federal de Ouro Preto, $M G$ (e-mail: anacastro@ufop.edu.br)
**** Departamento de Engenharia Mecânica, Universidade Federal de Ouro Preto, MG (e-mail: paulo.magalhaes@ufop.edu.br)

\begin{abstract}
Recent studies have shown that biofouling of ship's hull changes their hydrodynamics, increasing drag. It is estimated that losses can generate up to $20 \%$ increase in fuel consumption. As the scaling intensity increases, periodic cleaning should be performed. However, there is still no standard that determines the level or index of biofouling. In this work a virtual reality (VR) application is presented to simulate the inspection of biofouling conditions in ships by means of unmanned aerial vehicle (UAV). The digital surface models of biofouling is obtained by threedimensional photogrammetry. The biofouling index represents the intensity of fouling organisms in an area. The vehicle must fly over the vessel and record the biofouling, aiming to analyze the need to clean the hull. The work has as motivation the subsequent detection and identification of biofouling as represented in the simulated conditions.

Resumo: Estudos recentes comprovam que as bioincrustações em casco de navio alteram sua hidrodinâmica, aumentando as forças de arrasto. Estima-se que as perdas podem gerar um aumento de até $20 \%$ no consumo de combustível. Conforme a intensidade de incrustações aumenta, limpezas periódicas devem ser executadas. Entretanto, ainda não há um padrão que determina o nível ou índice de bioincrustação. Neste trabalho é apresentado uma aplicação de realidade virtual (VR) para simulação de inspeção das condições de bioincrustação em navios por meio de veículo aéreo não tripulado (VANT). Os modelos digitas das incrustações são obtidos por fotogrametria tridimensional. O índice de bioincrustação (IB) representa a intensidade de organismos incrustantes em uma área. O veículo deve sobrevoar a embarcação e registrar as bioincrustações, tendo como objetivo analisar a necessidade de limpeza do casco. O trabalho tem como motivação a posterior detecção e identificação de bioincrustação como representado nas condições simuladas.
\end{abstract}

Keywords: Virtual Reality; Biofouling; Photogrammetry; Ship inspection; Computer vision. Palavras-chaves: Realidade virtual; Bioincrustação; Fotogrametria; Inspeção de navio; Visão computacional.

\section{INTRODUÇÃO}

A navegação do transporte de minério aos portos dos consumidores pode atingir cerca $50 \%$ de seu custo. O tamanho dos navios e a quantidade de combustível gasto fazem com que o preço para movimentação do minério e manutenção dos navios seja alto Adland et al. (2018). Além do custo monetário, por utilizarem motores a combustão, os navios de carga têm uma participação muito significante na emissão de $\mathrm{CO}_{2}$ da parcela industrial.
Responsável por regulamentar e organizar o comércio marítimo, a International Maritime Organization (IMO) adotou em 2013 o conceito do Ship Energy Efficiency Management Plan (SEEMP), que propõe medidas operacionais para melhoria da eficiência energética das embarcações. A SEEMP é fundamentada em índices de design de eficiência energética, do inglês Energy Efficiency Design Index (EEDI), que inclui velocidade de operação, monitoramento e ajuste do motor, condições do casco e hélice, entre outros Santos (2017). 
Diversos estudos mostram que as incrustações de organismos vivos no casco, denominadas bioincrustações, podem implicar em um grande aumento na resistência à propulsão do navio em operação. Turan et al. (2016) e Santos (2017) estimam valores acima de $20 \%$ para esta perda, que pode variar com o nível de incrustação e velocidade de operação do navio. Em decorrência, serão grandes os impactos em custos e viabilidade econômica da frota, sendo urgente a necessidade de avaliar alternativas tanto para projeto de novos navios quanto para retrofit da frota atual a fim de manter a competitividade da produção e adequação às exigências de eficiência energética impostas pela IMO.

Atualmente, existem metodologias de controle da bioincrustação de organismos, como a aplicação de tintas antiincrustante e limpeza do casco e hélice realizada por mergulhadores em navios fundeados. A limpeza também pode ser efetuada em embarcações docadas em estado de manutenção, sem a necessidade de um trabalho submerso. O armador necessita analisar o custo da operação de limpeza em comparação com o custo gerado pelo aumento do consumo de combustível do navio, devido ao arrasto gerado pelas bioincrustações. Porém, não há na literatura uma definição clara da necessidade de limpeza quanto à intensidade de bioincrustação. Sendo assim, é elaborado no presente trabalho o índice de bioincrustação (IB), que representa uma proporcionalidade do volume incrustante em superfícies.

Quando o navio está fundeado, parte das incrustações presentes no casco que estavam submergidas são expostas. Dessa forma, é possível fazer uma inspeção visual da presença desses organismos no casco. Entretanto, o tempo de descarregamento, carregamento e espera do navio em porto geralmente não é suficiente para uma vistoria eficaz das condições do casco.

Existe como tecnologia emergente o uso de veículo aéreo não tripulado (VANT), tipicamente conhecido por drone, para vistoria de plantas industriais e equipamentos. Bem como, o uso de realidade virtual para realização de treinamento e simulações em um ambiente totalmente controlado, sem risco de danos à saúde dos operadores e de perda de bens materiais. A proposta do presente trabalho é conciliar ambas tecnologias para desenvolver uma solução inteligente de vistoria de casco de navio que determine o índice de bioincrustação.

Mesmo que as incrustações expostas ao ar livre representem apenas uma parcela da totalidade presente no navio, o seu estudo serve de sustentação para o próximo nível da pesquisa. Ou seja, primeiro pretende-se investigar as incrustações emergidas à superfície para então estudar a grande parte submersa. Para isso, seria necessário um veículo submarino operado remotamente equipado de sensores que atuam sobre condições diversas de luminosidade e visibilidade.

\section{OBJETIVOS}

Este trabalho tem como objetivo desenvolver um software de realidade virtual para simular um ambiente de detecção de bioincrustação em cascos de navios por meio de câmeras e sensores acoplados à VANTs.
A partir de um ambiente virtual, futuramente será possível explorar as possibilidades de operação de vistoria, que englobam os sensores a serem utilizados, métodos de identificação, tempo de operação, número de VANTs, rotas de vistoria, entre outros. A experiência de usuário com o software deverá trazer um retorno dessas possibilidades descritas, otimizando o objetivo final de mapear parte das bioincrustações presentes no casco de um navio de grande porte. Sendo assim, as possibilidades de operação analisadas e o treinamento prévio realizado pelos operadores resultará na redução de custos operacionais.

\section{FUNDAMENTAÇÃO TEÓRICA}

\subsection{Bioincrustação}

A bioincrustação marinha (ou biofouling) é definida por Gama et al. (2009) como um processo resultante da colonização ou do crescimento de bactérias, algas e/ou invertebrados sésseis sobre superfícies submersas. A bioincrustação é um grande problema presente na navegação, onde os organismos fixam em cais, plataformas, cascos e hélices de navios, boias, cabos, etc., causando danos econômicos e ambientais.

Segundo Davis et al. (1989), uma das principais bioincrustações presentes em navio, que possuem grande força de fixação nos cascos são os seres da Subclasse Thoracica, conhecidos como cracas. As cracas são crustáceos incrustantes de substratos duros, sejam eles naturais, por exemplo, recifes de corais ou artificiais (deques e embarcações). Algumas espécies possuem ampla distribuição geográfica e ampla tolerância ambiental e têm sido consideradas como espécies exóticas e invasoras por todos os continentes.

A capacidade de incrustar e as conchas, combinadas com a dispersão larval planctônica na maioria das espécies (via água de lastro), fornecem às cracas ferramentas adaptativas para invadir e incrustar em diversos ambientes. A capacidade de se aderir química e fisicamente das cracas dificulta a manutenção das embarcações e gera altos custos incluindo a limpeza do casco dos navios por equipes de mergulhadores. Assim, a incrustação das cracas em navios tem sido frequentemente reportada como uma das principais dificuldades em diminuir o atrito no casco dos navios (Kim et al., 2009).

Como exemplo de que problema também se estende à água doce, sendo um maiores casos de bioinvasão, o mexilhãodourado (Limnoperma fortunei) é um tipo de incrustação originária da Ásia. A espécie chegou à América do Sul provavelmente de modo acidental, pela troca de água de lastro de navios cargueiros. Em sua tese, Rico (2018) descreve os impactos gerados pela espécie invasora no Brasil, bem como em outros países. O autor analisa as perdas de carga gerada pela rugosidade equivalente da incrustação nas tubulações de hidrelétricas.

A variação da superfície devido incrustações presentes nos cascos da embarcação interferem no arrasto hidrodinâmico. Medir a geometria da superfície é importante para entender as características do fluxo. Segundo Rico (2018), as bioincrustações presentes no casco da embarcação podem ser representadas por um valor de coeficiente de rugosidade. Em que, selecionar um valor de coeficiente 
de rugosidade significa estimar a resistência ao escoamento exercida sobre o fluido.

Os autores Turan et al. (2016) demonstram a obtenção de uma superfície incrustada de cracas por meio de escaneamento 3D. Os modelos escaneados são impressos e analisados em testes de hidrodinâmica. Foi estimando uma diferença de $59,7 \%$ de potência efetiva do navio com velocidade de 20 nós e o casco $20 \%$ coberto de cracas.

Pelos danos que causam, as espécies exóticas invasoras são consideradas de alto risco para o bioma invadido. Estudos mostram que as invasões biológicas são a segunda maior causa de extinção de espécies, atrás apenas da destruição de habitats (Agudo-Padrón, 2008).

Portanto, é necessária a fiscalização dos cascos de navios em portos, assim como são requeridas tecnologias de inspeção visual para dar mais agilidade ao processo de identificação e posterior remoção deste bioinvasor.

\subsection{Realidade Virtual}

As palavras realidade e virtual separadas representam significados divergentes, como concreto e abstrato. No entanto, em seu livro, Ryan (2015) explica como esses dois termos juntos são utilizados para representar uma experiência ou simulação de uma realidade alternativa. A interação com o ambiente virtual é realizada em tempo real com o uso de técnicas e equipamentos computacionais que ajudem na ampliação do sentimento de presença do usuário neste ambiente. Esta sensação de presença é usualmente referida como imersão.

O termo "realidade virtual" vem do inglês virtual reality (VR), que foi definido por Pimentel and Teixeira (1993) como uma experiência totalmente interativa e imersiva gerada por um computador. Para uma experiência de VR completa, necessita-se de interfaces entre o usuário e o software onde se encontra o ambiente virtual. Atualmente já contamos com dispositivos de capacidade gráfica suficiente para criar a sensação de presença em um ambiente virtual.

Em 1838, o pesquisador britânico Charles Wheatstone deu início ao uso do estereoscópio para experiências de visualização tridimensional de paisagens. O método consiste basicamente da disposição de duas fotografias lado a lado, com uma pequena defasagem de posição. Então, por meio de lentes, as imagens são ampliadas logo à frente do observador. Esse método é utilizado até os dias de hoje. Atualmente existem diversos dispositivos de VR que projetam imagens estéreo por meio de um display eletrônico.

Com a capacidade de gerar imagens em alta resolução, os smartphones atuais podem ser utilizados como uma alternativa de baixo custo aos óculos de realidade virtual. O Google Cardboard é uma plataforma aberta de realidade virtual que disponibiliza em seu endereço eletrônico o projeto de seus óculos de VR para smartphones. Como plataforma de desenvolvimento em realidade virtual, o Unity é um ambiente de desenvolvimento de jogos 2D e 3D multiplataforma, permitindo a compilação para diversos dispositivos, como PC, Mac, Android e iOS. É baseado em programação intuitiva por blocos, ou "arrastar e soltar", aceitando scripts em linguagem de programação C\# Technologies (2018).
Para tornar um ambiente virtual ainda mais próximo da realidade, pode ser utilizado o recurso de escaneamento tridimensional (3D) para reconstruir virtualmente objetos e cenários reais. Por apresentar uma precisão elevada, a tecnologia mais empregada para o escaneamento 3D tem sido a triangulação a laser, efetuada por scanners. Entretanto, como alternativa de menor custo e com possibilidade de replicar cores e texturas, a fotogrametria vem ocupando espaço no mercado de tecnologias de escaneamento 3D.

\subsection{Fotogrametria}

A fotogrametria é definida como a ciência aplicada, a técnica e a arte de extrair de fotografias métricas, a forma, as dimensões e a posição dos objetos nelas contidos. É uma ferramenta bastante eficaz no processo de digitalização 3D a partir de fotografias. Para aplicações que exigem detalhamento, pode-se obter uma precisão com valores inferiores à $1 \mathrm{~mm}$.

A partir de modelos 3D é possível fazer testes e simulações em softwares de engenharia, ou replicar uma peça específica por exemplo. Como exemplo da eficácia dos recursos de fotogrametria, menciona-se o trabalho de Chung and Wang (2011). Em seu trabalho, os autores obtêm um modelo digital de superfície (MDS) de uma área de $6 \mathrm{~m}$ x $6 \mathrm{~m}$ do solo de cascalho exposto de um rio. Apenas com recurso de fotogrametria de pós-processamento, o desvio padrão das medidas foi de $2.2 \mathrm{~mm}$. A câmera utilizada foi uma Nikon D200 com uma lente $20 \mathrm{~mm}$ AF-S NIKKOR. Com este modelo, os autores propõem a possibilidade de análise de rugosidade da superfície.

Aplicações de VANTs em processos de monitoramento e vistoria aumentaram rapidamente nos últimos anos. Os VANTs equipados com câmeras e diversos outros sensores permitem que a vistoria seja realizada com maior eficácia e segurança, melhorando as condições de segurança para o operador. Em Burdziakowski (2018), é demonstrado a utilização de um VANT para a reconstrução 3D de um ambiente interno utilizando fotogrametria com processamento em tempo real.

\section{MATERIAIS E MÉTODOS}

O software foi desenvolvido em Unity, com configurações gráficas e compatibilidade específicas para a plataforma Android. O recurso de realidade virtual para Google Cardboard é disponibilizado por padrão nas configurações de compilação do Unity. Esse recurso simplifica a transformação do software elaborado em um computador, com uma única perspectiva de visualização para uma visão estéreo adaptada às lentes, criando a sensação de profundidade em VR.

Para a experiência com realidade virtual foi adquirido o VR Box, um óculos de realidade virtual de baixo custo que funciona como um suporte para o smartphone transmitir as imagens ampliadas ao usuário, assim como um Google Cardboard. O VR Box acompanha de um controle bluetooth, para que o usuário possa interagir com o ambiente virtual. O smartphone utilizado para testes foi um Xiaomi Redmi Note 6 Pro, equipado com o chipset Qualcomm Snapdragon 636 e sistema operacional Android. O usuário 
controla o veículo por meio do joystick, que já possui componentes de controle implementados nativamente.

\subsection{Determinação do índice de bioincrustação}

Foram realizadas buscas no banco de dados do Web of Science, no período de 1945 até 2019, utilizando o termo de busca "índice AND (bioincrustação OR incrustação)". Como resultado, apenas um artigo de 2017 foi encontrado, porém não tem relação com bioincrustação marinha. Buscando pelo termo exato "biofouling index", nenhum documento é encontrado. Quando a busca é feita com as palavras separadas "biofouling AND index", são encontrados 147 documentos científicos. Filtrando pelos mais recentes (de 2015 a 2019) são exibidos 53 artigos, sendo "silt density index" (SDI) o termo mais relacionado à incrustação marinha, que está presente em 3 dos 53 resumos.

O SDI é aplicado para medir a capacidade que uma amostra de água tem em incrustar determinada superfície, sendo mais empregado em sistemas de filtragem. Entretanto, para o problema abordado no presente trabalho, não há como mensurar este índice em um sistema aberto e variável como o oceano. Desta forma, é apresentado nessa seção uma determinação do índice de bioincrustação (IB) presente em cascos de navio.

Partindo do princípio de que a intenção do IB é de ser visualmente perceptível e aplicável em sistemas inteligentes com recursos de visão computacional, são considerados parâmetros com nível de precisão que podem ser mensurados a uma distância mínima para efetuar o sensoriamento, não envolvendo análise química ou microscópica. Também levando em consideração o tamanho dos navios mineraleiros (maior que $300 \mathrm{~m}$ de comprimento) e recursos disponíveis, a obtenção dos parâmetros deve ser relativamente rápida em relação ao tempo disponível para a inspeção.

Como a precisão de sistemas de escamento e digitalização tridimensional dependem da distância de atuação e da área de captura, recomenda-se utilizar áreas amostrais de tamanho fixo, preservando o padrão de detalhamento das amostras. Com o auxílio de sistemas inteligentes de mapeamento, as áreas analisadas não devem coincidir. Naturalmente, quanto maior a área de base e o número de amostras, maior será a representatividade do índice calculado da região.

Utilizando a área de parâmetro base, densidade e espessura de incrustação são analisados. Em um navio, será encontrado intensidades de incrustação conforme a profundidade em que o casco é submerso, bem como ao fluxo de água corrente em cada região. Com uma área definida, pretendese obter amostras de densidade e espessura da incrustação. Dessa forma, o índice de bioincrustação de uma região total (IB) é calculado pela média das amostras de IB $\left(I B_{i}\right)$ de um total de $n$ amostras, ou seja

$$
I B=\frac{\sum_{i=1}^{n} I B_{i}}{n}
$$

Sendo $I B_{i}$ definido por

$$
I B_{i}=D \times E
$$

Em que: D representa a densidade de incrustação, calculada pela área incrustada sobre a área amostral $\left(A_{i}\right) ; \mathrm{E}$ equivale à espessura média em $\mathrm{mm}$. $\mathrm{O}$ nível de representatividade de IB $\left(R_{I B}\right)$ pode ser calculado por

$$
R_{I B}=\frac{n \times A_{i}}{A_{t}}
$$

Em que $A_{i}$ é a área amostral e $A_{t}$ representa a área da região de interesse de determinação do índice.

Definidos a área total de interesse a ser inspecionada, os sensores e equipamentos a serem utilizados, o primeiro passo para determinar o IB é a área amostral. Esse valor pode variar com a capacidade de resolução dos sensores ópticos e de outras tecnologias e técnicas empregadas.

No presente trabalho é realizado uma simulação em VR com um VANT, entretanto os sensores a serem utilizados ainda estão em análise. Com base na revisão bibliográfica apresentada, estima-se que por meio da fotogrametria e auxílio de feixes de laser pode-se obter uma reconstrução tridimensional de parte do casco com precisão elevada e em tempo eficaz.

\subsection{Implementação do modelo $3 D$ de incrustação obtido por fotogrametria}

O modelo digital de bioincrustação foi gerado por meio de fotogrametria tridimensional de uma incrustação artificial de mexilhão-dourado (Figura 1). Utilizando o software de fotogrametria 3DF Zephyr da 3DFLOW, foram processadas 42 fotografias de alta resolução capturadas com uma câmera Canon EOS 60D. Foi utilizado o método de captura radial com duas alturas angulares, mantendo o objeto e iluminação fixos e efetuando a captura de imagens a uma distância de aproximadamente $60 \mathrm{~cm}$.

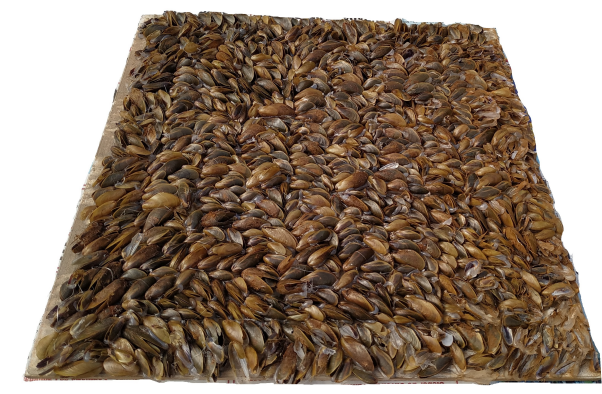

Figura 1. Incrustação artificial de mexilhão-dourado em uma cerâmica de 304 x 304 mm.

A malha obtida do processo de fotogrametria apresentou um alto nível de detalhamento, em que os contornos e algumas das concavidades dos mexilhões foram bem reconstruídos. No entanto, para a aplicação fluir bem em uma plataforma Android com um poder de processamento não tão elevado em comparação a um desktop, a malha gerada precisou ser cortada e simplificada. Para o corte, foi selecionando a região de interesse (meio da amostra), reduzindo o número de faces de 524.209 para 217.148. A simplificação da malha foi processada no software Open Source Meshlab, com o método "Simplification quadric edge collapse decimation (with texture)". O número de faces foi reduzido de 217.148 para 50.000 , com uma textura de resolução 4096 x 4096 pixels. 
A malha final no formato ".obj" foi importada ao projeto do Unity sem qualquer necessidade de conversão. Foram dispostas 5 cópias da reconstrução alcançada, variando as dimensões de área, densidade e espessura. Com a malha tratada, houve um aumento muito perceptível na fluidez do aplicativo final, porém a qualidade gráfica não sofreu grandes perdas, conforme a Figura 2. Observe que as concavidades do mexilhão foram preservadas.

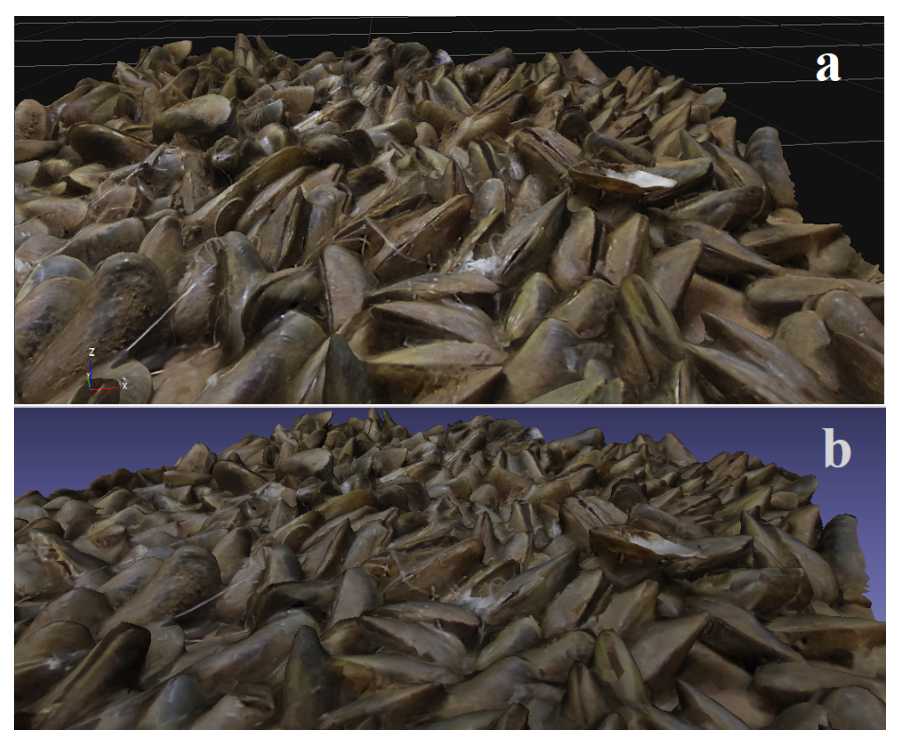

Figura 2. (a)Malha original cortada com 217.148 faces (b)Malha simplificada com 50.000 faces.

\subsection{Cenário virtual e imersão}

O cenário é composto por um VANT quadricóptero com controle já implementado, que deve gravar as áreas amostrais de bioincrustação em uma embarcação genérica fundeada (Figura 3). O usuário deve manipular o veículo em uma parte específica da embarcação e fazer a captura dos dados. Um retículo localizado ao centro da tela interage com as incrustações, de tal forma que exibe os status de busca, identificação e se a área já foi vistoriada.

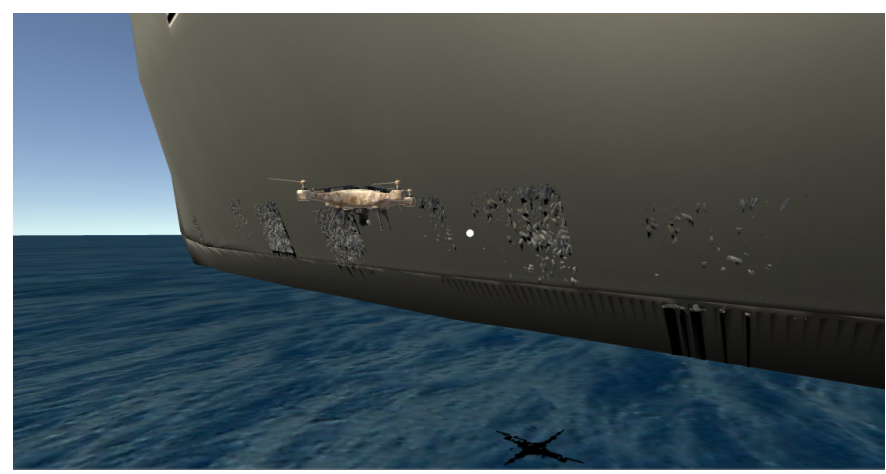

Figura 3. Cenário de testes em realidade virtual

As incrustações são formadas por variações do modelo $3 \mathrm{D}$ da mexilhão-dourado obtido por fotogrametria, apresentado na seção anterior. Para que ocorra a identificação e registro, o retículo interativo deve ser posicionado de forma que coincida com a incrustação desejada e então, aguardar alguns segundos até que programa indique a conclusão do processo. As áreas identificadas serão adicionadas a um relatório, contendo as informações de espécie predominante, e índice de bioincrustação.

\section{RESULTADOS PRELIMINARES}

O projeto resultou na versão final do aplicativo para smartphone, já com todos os objetos e funções implementadas. O sistema simulado de identificação funcionou de modo correto, registrando os parâmetros do índice de bioincrustação, bem como a espécie predominante em cada área registrada. Na Figura 4 está representado o estado de busca, em que o veículo não se encontra em distância mínima suficiente para efetuar a varredura de uma nova área amostral. Ao lado esquerdo é possível notar que três $A_{i}$ já foram registradas, que estão marcadas de verde.

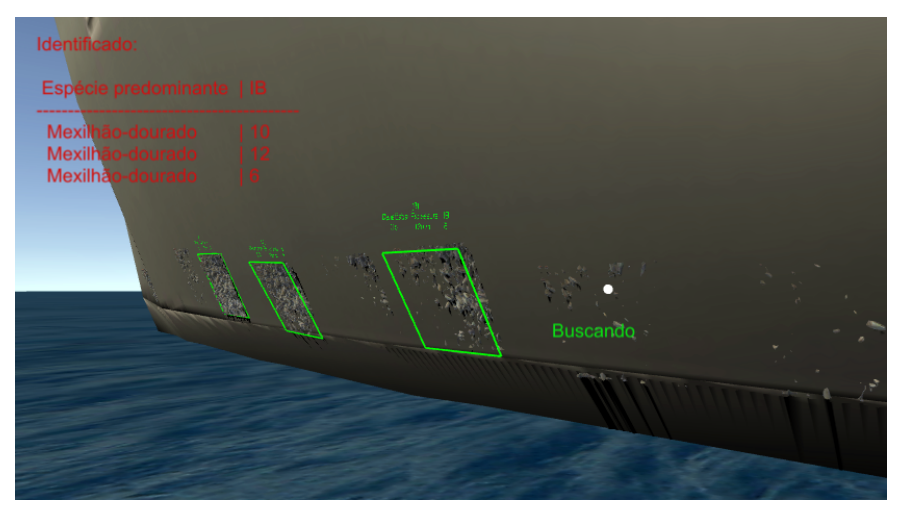

Figura 4. Estado de busca. Em vermelho: registro das áreas amostrais contendo espécie predominante e IB.

Na Figura 5, pode-se visualizar o estado de identificação e o registro visual dos parâmetros de IB. Foi considerado uma $A_{i}$ fixa de $1 m^{2}$, que é marcada com um retângulo verde. Os valores de densidade, espessura e IB são exibidos acima de cada $A_{i}$, considerando um afastamento de pelo menos uma vez a largura de cada área.

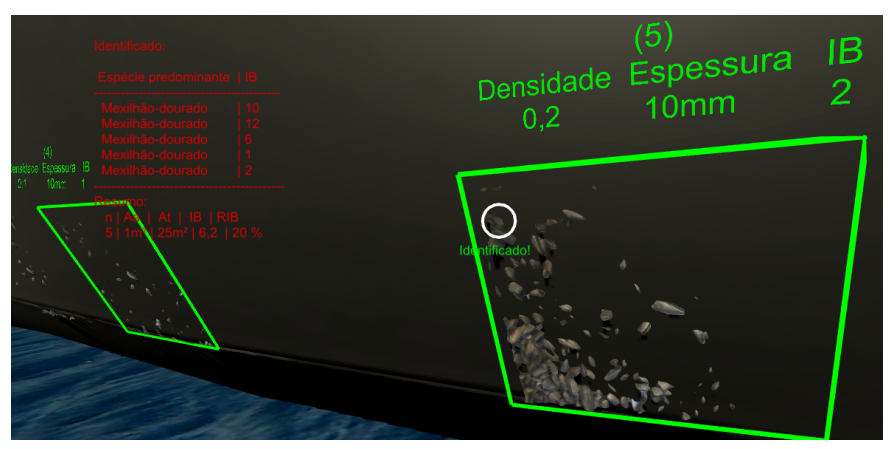

Figura 5. Estado de identificação e o registro visual dos parâmetros de IB.

Ao final da vistoria, o relatório emite um resumo conforme a Figura 5. São exibidos o número de amostras (n), área amostral utilizada $\left(A_{i}\right)$, área total considerada $\left(A_{t}\right), I B$ e $R_{I B}$.

O aplicativo, ao ser instalado no smartphone, já fica pronto para o uso, bastando apenas rodar sua aplicação e posiciona-lo no VR BOX, que foi utilizado no desenvolvimento do trabalho. 


\section{CONSIDERAÇÕES FINAIS}

O objetivo de desenvolver uma plataforma em realidade virtual que simula a vistoria de bioincrustações em casco de navio foi atingido. Por métodos de deteç̧ão e processamento de imagem ainda não definidos, o sistema de câmeras e sensores deve ser capaz de replicar a metodologia abordada neste trabalho. A imersão em VR possibilitou que o usuário tenha a percepção de como seria realizada a vistoria real. Isso é importante para a análise de implementação de um equipamento ou técnica ao projeto real, que antes pode ser simulada em VR.

Vale ressaltar, que o presente trabalho demonstrou a simulação em realidade virtual da metodologia de vistoria, mas não apresentou definições de equipamentos e técnicas específicas de aquisição de dados. O desenvolvimento do índice de bioincrustação aplicável à engenharia é o grande desafio abordado até o momento.

Por ser uma simulação, o custo de implementação de métodos de detecção e de registro dos seres incrustantes é muito menor comparado a treinamentos operacionais reais. Assim como é mais acessível avaliar a experiência de usuário durante a vistoria realizada em VR, reduzindo assim quaisquer riscos relacionados a danos ou acidentes em operação.

Em trabalhos futuros serão estudadas técnicas de visão computacional, que englobam a identificação visual de espécies incrustantes, medição de espessura, área incrustada e rugosidade da superfície. Dessa forma, será possível definir uma escala representativa para o índice de bioincrustação (IB) que foi elaborado. Essas técnicas poderão ser analisadas no software desenvolvido. Em uma aplicação real de vistoria, ainda há a possibilidade de adaptação do software em VR para realidade aumentada, auxiliando o operador do veículo equipado de sensores a executar as atividades de registro, assim como foi feito em simulação.

\section{AGRADECIMENTOS}

Universidade Feral de Ouro Preto, Instituto Tecnológico Vale.

\section{REFERÊNCIAS}

Adland, R., Cariou, P., Jia, H., and Wolff, F.C. (2018). The energy efficiency effects of periodic ship hull cleaning. Journal of Cleaner Production, 178, 1-13.

Agudo-Padrón, A.I. (2008). Vulnerabilidade da rede hidrográfica do estado de santa catarina, sc, ante o avanço invasor do mexilhão-dourado, limnoperna fortunei (dunker, 1857). Revista Discente Expressões Geográficas, 4(4), 75-103.

Burdziakowski, P. (2018). Uav design and construction for real time photogrammetry and visual navigation. In 2018 Baltic Geodetic Congress (BGC Geomatics), 368372. IEEE.

Chung, J.T. and Wang, C.K. (2011). DSM measurement for exposed gravel surface using close-range photogrammetry with multi-scale images. International Geoscience and Remote Sensing Symposium (IGARSS), 471-474. doi:10.1109/IGARSS.2011.6049167.
Davis, A.R., Targett, N.M., McConnell, O.J., and Young, C.M. (1989). Epibiosis of marine algae and benthic invertebrates: natural products chemistry and other mechanisms inhibiting settlement and overgrowth. In Bioorganic marine chemistry, 85-114. Springer.

Gama, B., Pereira, R., and Coutinho, R. (2009). Bioincrustação marinha. Biologia Marinha, 2nd edn. Interciência, Rio de Janeiro, 299-318.

Kim, D., Jung, S., Sohn, J., Kim, H., and Lee, S. (2009). Biocide application for controlling biofouling of swro membranes - an overview. Desalination, 238(1-3), 4352.

Pimentel, K. and Teixeira, K. (1993). Virtual reality through the new looking glass. CUMINCAD.

Rico, E.A.M. (2018). Influência da taxa de infestação de Limnoperna Fortunei no aumento da perda de carga em sistemas hidráulicos. Thesis, Universidade Federal de Minas Gerais.

Ryan, M.L. (2015). Narrative as virtual reality 2: Revisiting immersion and interactivity in literature and electronic media, volume 2. JHU Press.

Santos, R.F.D.e.M.d. (2017). Planejamento de manutenção no casco e hélice de navios por meio da análise de dados. Ph.D. thesis, Universidade Federal do Rio de Janeiro.

Technologies, U. (2018). Unity multiplataform main page. https://unity3d.com/pt/unity/ features/multiplatform. Accessed: 2018-14-12.

Turan, O., Demirel, Y.K., Day, S., and Tezdogan, T. (2016). Experimental determination of added hydrodynamic resistance caused by marine biofouling on ships. Transportation Research Procedia, 14, 1649-1658. 